\title{
Eosinophilia in Patients Infected with Human Immunodeficiency Virus
}

\author{
Andrew Chou, MD and Jose A. Serpa, MD, MS \\ ${ }^{1}$ Section of Infectious Diseases, Department of Medicine, Baylor College of Medicine, Houston, \\ Texas
}

\begin{abstract}
Eosinophilia is not uncommonly encountered in patients infected with human immunodeficiency virus (HIV); particularly at initiation of care or among those with advanced disease. The clinical manifestation most commonly associated with eosinophilia in this patient population is skin rash. Management of these patients is challenging due to a paucity of data evaluating diagnostic testing and therapeutic strategies. Patients born in or with significant travel to parasite-endemic countries are more likely to have tissue-invasive helminthes, such as Strongyloides or Schistosoma. Patients without such risk factors are unlikely to have parasitic infections and frequently will have selfresolution of eosinophilia. When a detailed history, physical exam and diagnostic work-up is unrevealing, we sometimes consider empirical therapy with ivermectin. Praziquantel may also be considered for those at risk for schistosomiasis.
\end{abstract}

\section{Keywords}

eosinophilia; human immunodeficiency virus; parasites; management; presentation; prognosis

\section{Introduction}

Eosinophilia is not uncommon in patients infected with human immunodeficiency virus (HIV), but clinical management is challenging due to a paucity of high-quality data supporting a standard approach for diagnostic testing or management of these patients [1-3]. Limitations of the existing studies include variation in geography, patient populations, thresholds for defining eosinophilia, use of highly active antiretroviral therapy (HAART), clinical characteristics and outcomes examined. In addition, the majority of these studies were retrospective single-center experiences. Although an optimal diagnostic and therapeutic approach for HIV-infected patients presenting with eosinophilia has not been well defined, here we summarize the existing data to assist in the management of these patients.

Corresponding author: Jose A. Serpa, One Baylor Plaza, BCM 286, Houston, TX 77030, USA. jaserpaa@ bcm.edu. Conflict of interest: Neither of the authors reports any relevant potential conflicts of interest.

Human and Animal Rights and Informed Consent: This article does not contain any studies with human participants or animals performed by any of the authors. 


\section{Pathophysiology and Etiology of Eosinophilia}

Eosinophils are terminally differentiated granulocytes that mature in the bone marrow, are released into the blood briefly (half-life $\sim 18$ hours), and migrate to peripheral tissues, such as the gastrointestinal tract [4]. Traditionally, the presence of eosinophilia, regardless of the HIV status of the patient, has been associated with an underlying disease process. In developing countries, eosinophilia is most commonly associated with infectious processes (Table 1) such as helminth-associated and fungal conditions, while in developed countries, eosinophilia is most commonly associated with allergic conditions[1-3,5,6].

In the presence of certain parasitic infections, eosinophils are recruited to the site of the parasitic infection and promote a Th2 immune response. When parasites migrate through tissue, they are directly exposed to the host's immune cells and produce local and peripheral eosinophilia $[4,5]$. Therefore, patients infected with tissue invasive helminthes and burrowing ectoparasites commonly present with eosinophilia. Parasites that are exclusively intraluminal (e.g. adult tapeworms, protozoa) or contained in cystic structures (e.g. hydatid cyst, neurocysticercosis) are unlikely to cause eosinophilia unless there is a disruption in the barrier between the parasite and host $[4,5]$.

Coccidioidomycosis and aspergillosis are two well-known fungal causes of eosinophilia[4,5,7]. Infections with Coccidioides spp. have been reported to cause extreme eosinophilia, and eosinophilia may be associated with disseminated disease. It is unclear what role eosinophils play in coccidioidomycosis $[4,5,8]$. One specific form of aspergillosis, allergic bronchopulmonary aspergillosis (ABPA), is associated with eosinophilia. ABPA is an inflammatory airway disease that is promoted by the presence of Aspergillus spp. in the lungs, rather than a tissue invasive process. Recent data have shown that the allergic airway inflammation is mediated by fungal-derived proteinases, which are found not only in Aspergillus spp., but also in other environmental fungi, most notably Penicillium and Curvularia spp. [9]. Histoplasmosis and tuberculosis can cause eosinophilia indirectly when they cause adrenal insufficiency, but they are unlikely to cause eosinophilia in other contexts. HIV itself is thought to cause eosinophilia due a Th1-Th2 shift $[6,10]$.

In addition to infectious causes, patients infected with HIV may have eosinophilia due to non-infectious causes such as eosinophilic pustular folliculitis, drug reactions due to HAART or antimicrobials, malignancies, hypereosinophilic syndromes, toxins among others. Of note, virtually all HIV antiretroviral agents can cause rash, but very few are associated with eosinophilia, such as drug reaction with eosinophilia and systemic symptoms (DRESS), which has been well-described with nevirapine and raltegravir [1113].

Other causes of eosinophilia, not necessarily seen more frequently in patients with HIV infection, include: solid organ tumors, hematologic malignancies, primary immunodeficiencies, and autoimmune diseases. 


\section{Clinical Presentation and Prognosis of Patients with Eosinophilia and HIV Infection}

There is no universally agreed upon definition for peripheral eosinophilia, but it is commonly defined as an absolute eosinophil count of greater than 400-500 cells/microliter. Eosinophilia is encountered in as high as $28 \%$ of patients with HIV infection [6]. In developed countries, higher rates of eosinophilia have been seen in patients initiating HIV care, and those with advanced HIV disease. Early studies prior to the introduction of HAART found that CD4+ T-cell count below 200 cells/microliter was associated with eosinophilia, along with possibly advanced disease (CDC disease stage C) and primary Pneumocystis carinii pneumonia prophylaxis [14]. Subsequent studies confirmed these findings. In a study of HAART-naïve HIV-infected patients found that CD4+ T-cell count below 50 cells/microliter was associated with eosinophilia [6]. Another study in foreignborn HIV-infected patients who were receiving HAART found that CD4+ T-cell count below 200 cells/microliter was associated with eosinophilia [1,14]. Skin rash is also commonly associated with eosinophilia, although a definitive (i.e. biopsy-proven) diagnosis is often not made [6]. A study of patients with advanced HIV during the pre-HAART era found that pruritic skin rashes, in particular eosinophilic pustular folliculitis, were associated with eosinophilia [15].

\section{Patients in Developed Countries with Epidemiological Risk Factors for Parasitic Infections}

Among patients with eosinophilia and HIV infection, the etiology of eosinophilia varies based on patient demographics. In studies of foreign-born patients with HIV infection living in developed countries, eosinophilia was associated with positive serology for Schistosoma and Strongyloides; whereas Chagas disease, neurocysticercosis, and other intestinal parasitic infections were not associated with eosinophilia [1,14-17]. The CD4+ T-cell count and length of time since immigration did not differ between those infected and uninfected with parasites, likely because the implicated helminthes, Schistosoma and Strongyloides, can cause chronic infection due to a prolonged life span and autoinfection, respectively [16,17].

\section{Patients in Developed Countries without Epidemiological Risk Factors for Parasitic Infections}

In studies that did not specifically enroll patients from parasite endemic countries, the incidence of parasitic infections was very low and not different from control groups $[6,15]$. One of these studies followed HAART-naïve patients at a clinic where approximately $25 \%$ of patients were Hispanic [18], found that $73.1 \%$ of cases had resolution of eosinophilia by the end of the study (mean follow-up 2.8 years). The study was unable to identify immunologic or virologic factors associated with resolution of eosinophilia, including initiation of HAART, HIV viral suppression, or CD4+ T-cell count [6]. These data suggest that eosinophilia in patients infected with HIV without travel to parasite endemic countries may have eosinophilia due to non-infectious etiologies and appear to have a relatively favorable prognosis. 


\section{Management of Eosinophilia}

Management of HIV infected patients with eosinophilia is challenging particularly when efforts are made to screen foreign-born persons for parasitic infections. Multiple diagnostic methods exist and each has varying sensitivity, specificity, and availability [16].

In our practice, we suggest obtaining a detailed history and physical, with particular attention to certain organ-systems such as the skin, gastrointestinal tract, and genitourinary tract. In addition, we recommend collecting a meticulous medication and social history. Patients with advanced HIV disease presenting with acute illness and eosinophilia should be evaluated for disseminated opportunistic infections, including histoplasmosis and tuberculosis. Patients with recent initiation of HAART or antimicrobial agents should be evaluated for drug reaction to these agents.

For patients with persistent eosinophilia born in or with significant travel to countries where parasitic infections are endemic, and who have an unrevealing diagnostic work-up or will be immunosuppressed (e.g. steroids), we consider empirical treatment with ivermectin to cover most nematodes if there is no evidence of filariasis; and for those at risk for schistosomiasis (e.g. having lived in or traveled to Africa, Asia, or certain regions of Central and South America), we also consider praziquantel. These parasites can cause chronic infections, and significant morbidity; however, the majority of them can be easily treated with antiparasitics prior to progressing to severe complications [1].

\section{Summary}

Eosinophilia in patients with HIV infection is challenging, and not infrequently encountered. Eosinophilia is associated with a variety of conditions including allergic processes, parasitic and fungal infections, advanced HIV disease, and adrenal insufficiency among others. For patients from parasite-endemic countries, Schistosoma and Strongyloides are the most likely parasites to be associated with eosinophilia, regardless of CD4+ T-count or time since immigration. Commercially available diagnostic tests for many parasites are suboptimal, and empiric treatment with ivermectin may be recommended in certain patient populations. Those from regions where Schistosoma spp. are endemic may also benefit from empirical praziquantel. As more sensitive diagnostic tests for parasites are developed, further studies will be needed to define the real burden of these infections in patients with eosinophilia and HIV infection.

\section{Acknowledgments}

Funding: A.C. is funded by the NIH (grant T32AI055413).

\section{References}

1**. Sivaram M, White A, Radcliffe KW. Eosinophilia: clinical significance in HIV-infected individuals. International Journal of STD \& AIDS. 2012; 23:635-8. Study examining the relationship between eosinophilia and parasitic infections in patients with HIV. [PubMed: 23033516]

2. Cohen AJ, Steigbigel RT. Eosinophilia in patients infected with human immunodeficiency virus. J Infect Dis. 1996; 174:615-8. [PubMed: 8769622] 
3. Adewuyi JO, Coutts AM, Latif AS, Smith H, Abayomi AE, Moyo AA. Haematologic features of the Human Immunodefiency Virus (HIV) infection in adult Zimbabweans. Central African Journal of Medicine. 1999:45. [PubMed: 10444900]

4. Rosenberg HF, Dyer KD, Foster PS. Eosinophils: changing perspectives in health and disease. Nat Rev Immunol. 2012; 13:9-22. [PubMed: 23154224]

5*. Mejia R, Nutman TB. Evaluation and differential diagnosis of marked, persistent eosinophilia. Semin Hematol. 2012; 49:149-59. Differentiates which parasitic infections typically cause eosinophilia. [PubMed: 22449625]

6**. Mohajer Al M, Villarreal-Williams E, Andrade RA, Giordano TP, Serpa JA. Eosinophilia and Associated Factors in a Large Cohort of Patients Infected with Human Immunodeficiency Virus. South Med J. 2014; 107:554-8. Study examining the factors associated with eosinophilia in patients with HIV. Most patients had resolution of eosinophilia. [PubMed: 25188618]

7. Fabre V, Beiting DP, Bliss SK, Gebreselassie NG, Gagliardo LF, Lee NA, et al. Eosinophil deficiency compromises parasite survival in chronic nematode infection. J Immunol. 2009; 182:1577-83. [PubMed: 19155506]

8. Harley WB, Blaser MJ. Disseminated coccidioidomycosis associated with extreme eosinophilia. Clin Infect Dis. 1994; 18:627-9. [PubMed: 8038321]

9. Porter P, Polikepahad S, Qian Y, Morgan Knight J, Lu W, Tai WMT, et al. Respiratory tract allergic disease and atopy: experimental evidence for a fungal infectious etiology. Med Mycol. 2011; 49:S158-63. [PubMed: 20807032]

10. Arndt MB, John-Stewart G, Richardson BA, Singa B, van Lieshout L, Verweij JJ, et al. Impact of Helminth Diagnostic Test Performance on Estimation of Risk Factors and Outcomes in HIVPositive Adults. PLoS ONE. 2013; 8:e81915. [PubMed: 24324729]

11. Wongkitisophon P, Vachiramon V, Chanprapaph K, Rattanakaemakorn P. Six-year Retrospective Review of Drug Reaction with Eosinophilia and Systemic Symptoms. Acta Derm Venerol. 2012; 92:200-5. [PubMed: 22002792]

12. Yee BE, Nguyen NH, Lee D. Extensive pulmonary involvement with raltegravir-induced DRESS syndrome in a postpartum woman with HIV. BMJ Case Reports. 201410.1136/bcr-2013-201545

13. Perry MEO, Almaani N, Desai N, Larbalestier N, Fox J, Chilton D. Raltegravir-induced Drug Reaction with Eosinophilia and Systemic Symptoms (DRESS) syndrome - Implications for clinical practice and patient safety. International Journal of STD \& AIDS. 2013; 24:639-42. [PubMed: 23970584]

14. Tietz A, Sponagel L, Erb P, Bucher H, Battegay M, Zimmerli W. Eosinophilia in patients infected with the human immunodeficiency virus. Eur J Clin Microbiol Infect Dis. 1997; 16:675-7. [PubMed: 9352261]

15. Skiest DJ, Keiser P. Clinical significance of eosinophilia in HIV-infected individuals. Am J Med. 1997; 102:449-53. [PubMed: 9217641]

16. Hochberg NS, Moro RN, Sheth AN, Montgomery SP, Steurer F, McAuliffe IT, et al. High prevalence of persistent parasitic infections in foreign-born, HIV-infected persons in the United States. PLoS Negl Trop Dis. 2011; 5:e1034. [PubMed: 21532747]

$17^{* *}$. Costiniuk CT, Cooper CL, Doucette S, Kovacs CM. Parasitic disease screening among HIV patients from endemic countries in a Toronto clinic. Can J Infect Dis Med Microbiol. 2012; 23:23-7. Study of the prevalence of parasitic infections in patients with HIV from parasiteendemic countries. [PubMed: 23450241]

18. Poon KK, Dang BN, Davila JA, Hartman C, Giordano TP. Treatment outcomes in undocumented Hispanic immigrants with HIV infection. PLoS ONE. 2013; 8:e60022. [PubMed: 23555868] 


\section{Table 1}

\section{Infectious Causes of Eosinophilia}

\begin{tabular}{|l} 
Helminthes \\
Nematodes (roundworm) \\
Angiostrongyloidiasis \\
Ascariasis \\
Filarial infections (Brugia, Wuchereria, Loa loa, Mansonella, Onchocerca) \\
Gnathostomiasis \\
Hookworms \\
Visceral larva migrans (Toxocara, Baylisascariasis) \\
Strongyloidiasis \\
Trichinosis \\
Trematodes (flukes) \\
Clonorchiasis \\
Fascioliasis \\
Fasciolopsiasis \\
Opisthorchiasis \\
Paragonimiasis \\
Schistosomiasis \\
Ectoparasites \\
Myiasis \\
Scabies \\
\hline Coccidioidomycosis \\
Allergic bronchopulmonary aspergillosis \\
\end{tabular}

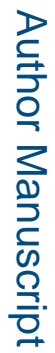

